\title{
Caring for COVID's Most Vulnerable Victims: a Safety-Net Hospital Responds
}

\author{
Miriam Komaromy, $\mathrm{MD}^{1,2}{ }^{(0)}$, Miriam Harris, MD, MSc ${ }^{1,2,3}$, Rob M. Koenig, MBA ${ }^{4}$, \\ Mary Tomanovich, $M A^{7}$, Glorimar Ruiz-Mercado, $M D^{7,5}$, and Joshua A. Barocas, $\mathrm{MD}^{7.5}$
}

'Grayken Center for Addiction, Boston Medical Center, Boston, MA, USA; ${ }^{2}$ Section of General Internal Medicine, Department of Medicine, Boston University, Boston, MA, USA; ${ }^{3}$ Clinical Addiction Research and Education (CARE) Unit, Boston University, Boston, MA, USA; ${ }^{4}$ Boston Medical Center, Boston, MA, USA; ${ }^{5}$ Section of Infectious Diseases, Department of Medicine, Boston University, Boston, MA, USA.

J Gen Intern Med 36(4):1006-10

DOI: $10.1007 / \mathrm{s} 11606-020-06499-2$

(c) Society of General Internal Medicine 2021

\section{BACKGROUND}

People experiencing homelessness (PEH) are at increased risk of infection from COVID-1 $19^{1-3}$ and recommended infection control measures are often not feasible. ${ }^{4}$ Frequent handwashing is difficult, shelters are crowded, and physical distancing is not possible; beds often have no barriers between them and are located in large rooms. Personal protective equipment (PPE) may not be available for guests or shelter staff. When COVID-19 infection occurs in PEH, they are often unable to isolate at home and may lack familial supports. These patients need help in order to recuperate, and it is essential that they isolate in order to reduce the risk of transmitting COVID-19 infection. ${ }^{5}$

PEH have higher rates of substance use disorders (SUDs) and mental health disorders than the general population. ${ }^{6}$ SUDs pose special challenges during the COVID-19 epidemic. $^{7}$ Harm reduction approaches traditionally rely on in-person interactions (e.g., mobile outreach) ${ }^{8}$ to build relationships and disseminate supplies. The COVID-19 physical distancing mandate disrupts programs' normal operations, risking greater incidence of complications like overdose. In isolation or quarantine settings, people with SUDs are at risk of withdrawal while confined without access to substances or treatment.

Boston Medical Center (BMC) has served as a safety-net hospital in Boston since its founding in 1855. Approximately 9\% of patients admitted to BMC are experiencing homelessness. As a result, BMC leaders prioritized addressing the unique needs of this vulnerable population.

\section{AIM}

BMC created a COVID Recuperation Unit (CRU) site to provide a safe and supportive place for PEH to isolate and

Received September 2, 2020

Accepted December 17, 2020

Published online January 19, 2021 receive care for COVID-19 symptoms, SUDs, and mental health disorders. The CRU may provide a practical model for municipalities to provide care for PEH who are COVID19 infected and may inform future pandemic-planning efforts.

\section{SETTING}

The first Massachusetts case of COVID-19 was reported in Boston on February 1, 2020. ${ }^{9}$ In early March 2020, addiction and infectious disease specialists at BMC began conversations with BMC leadership about the need to create capacity for PEH with COVID-19 to isolate and receive care, even if they did not require hospitalization.

In mid-March, the Boston Public Health Commission (BPHC) initiated daily meetings with leaders from public health and community-based organizations that provide care for PEH in Boston, including the Boston Department of Neighborhood Development and Bureau of Recovery Services, BMC, community harm reduction programs, leaders of large Boston shelter programs (including Pine Street Inn, St. Francis House, Rosie's Place, and BPHC shelters), and Boston Health Care for the Homeless Program, to forecast care needs and develop possible alternative care sites.

In late March, screening revealed high prevalence of COVID-19 infection in shelters. It became clear that larger facilities with expanded capacity were needed for PEH who were infected with COVID-19 in order to prevent rampant viral transmission. Within BMC, clinical leaders raised concerns about diverting workforce from staffing inpatient and intensive care units to staffing a COVID Recuperation Unit during the predicted surge in COVID-19 hospitalizations. However, the hospital CEO advocated for BMC to address this public health need in Boston. Like many hospitals, BMC expected to reach maximum bed capacity, and leadership was motivated to identify alternate care sites for patients who needed isolation but did not need to occupy inpatient beds. On March 24, 2020, the Commonwealth of Massachusetts agreed to loan $\mathrm{BMC}$ a vacant hospital building two blocks from the BMC Emergency Department (ED) for the development of a COVID Recuperation Unit (CRU) to serve PEH. 


\section{PARTICIPANTS}

The CRU served patients who were COVID-infected and experiencing homelessness. Patients were eligible if they were 18 or older, had tested positive for COVID-19, and were referred to the CRU for care. They ranged from asymptomatic (and simply needing to complete isolation for infection control) through moderately symptomatic but not in need of hospital-level acute care.

\section{PROGRAM DESCRIPTION}

\section{Rapid Deployment Was Assisted by Crisis Status}

The CRU admitted its first patients on April 9, 2020. Between March 24 and April 9, several features of the crisis allowed for rapid implementation of the CRU. First, the Massachusetts Department of Public Health (DPH) licensure for the CRU was obtained under the Authorization and Guidelines for Use of Alternate Space for Treatment of Patients During the COVID19 2020 State of Emergency. ${ }^{10}$ This allowed the hospital to perform clinical care in unlicensed space not owned by BMC. Second, the CRU was classified as a medicalized shelter by DPH, and as a "bedded outpatient" unit by the Drug Enforcement Administration (DEA), avoiding the need to qualify for inpatient level of care and permitting medications to be prescribed on an outpatient basis, which allowed the CRU to operate without an inpatient pharmacy. Third, anticipation of emergency funding allowed for simplified processes and documentation, since billing was not required. Fourth, staffing was available in part because the Medical Center paused non-essential services. Fifth, the CRU utilized many of BMC's existing systems: for instance, clinical programs in BMC's Grayken Center for Addiction, such as addiction treatment programs and counseling/social work, came together to support clinical services. The CRU also leveraged BMC's preparations for the COVID-19 crisis, such as support from facility management, Information Technology, infection control, and admitting. BMC's development department received donations for the CRU including furniture, televisions, clothing, and prepackaged meals delivered three times per day.

\section{Description of the COVID Recuperation Unit}

Physicians and advanced practice providers, nurses, medical assistants, clinical social workers, harm reduction staff, case managers, and security and operations staff provided roundthe-clock staffing for the CRU (Table 1). All staff wore full PPE at all times. Because all patients were COVID-infected, patients were not required to wear PPE.

Patient referrals were made via phone call or fax to a CRU admissions phone line. The phone number was publicized via email to local hospitals, homeless service agencies, shelters, and sites that were providing COVID-19 testing to PEH. Referring agencies were encouraged to send patients with two weeks' supply of their medications. Patients self-
Table 1 Staffing Model at COVID Recuperation Unit

\begin{tabular}{lll}
\hline \hline Fixed staffing, 2 floors & Day FTE & Night FTE \\
MD & 1.00 & 1.00 \\
Operation director & 1.00 & 1.00 \\
Discharge planner & 1.00 & $\mathrm{n} / \mathrm{a}$ \\
Security staff & 4.00 & 4.00 \\
Variable staffing ratio, per patient & Day & Night \\
NP/PA & $1: 88$ & $\mathrm{n} / \mathrm{a}$ \\
RN /LPN & $1: 33$ & $1: 33$ \\
Behavioral Health provider & $1: 88$ & $\mathrm{n} / \mathrm{a}$ \\
(counselor, social worker) & & \\
Harm reductionist & $1: 88$ & $\mathrm{n} / \mathrm{a}$ \\
Nurse Tech/Medical Assistant & $1: 22$ & $1: 22$ \\
Operation staff & $1: 22$ & $1: 22$ \\
\hline
\end{tabular}

administered most medications, which they kept locked at their bedside.

Many patients who were admitted to the CRU had SUDs. While treatment was offered, it was not expected that all patients who used substances would be interested in engaging in treatment. (See Text Box 1 for details of the SUD treatment approach.)

Text Box 1. Managing substance use disorders in the COVID Recuperation Unit

\footnotetext{
Goal of SUD management

The goal was to help patients tolerate isolation in the facility. Required adaptations of usual practice, e.g., prescribing higher doses of buprenorphine to suppress craving, were sometimes necessary. Addiction specialists could also offer stimulant or benzodiazepine prescriptions for stimulant or benzodiazepine use disorders, to suppress craving and deter leaving the unit. Cigarettes, usually banned from hospitals, were provided upon request, and smoking was permitted in an external courtyard.

Routine assessment for withdrawal

Withdrawal was common because patients were confined suddenly. All patients were assessed for withdrawal risk upon admission, and withdrawal treatment was available 24 hours per day.

Addiction consultation

Some medical staff were uncomfortable managing SUDs. Changed telehealth regulations allowed addiction specialists to perform telehealth consults, including initiating buprenorphine/methadone.

Methadone

Initially, methadone was obtained via take-home doses for patients who were already enrolled in outside opioid treatment programs (OTPs).This was operationally burdensome. Buprenorphine was also contraindicated for some patients with opioid withdrawal or use disorder (if, for instance, they had been using illicitly obtained methadone or longer-acting fentanyl analogs, or they preferred methadone treatment). Therefore, medical staff consulted with the DEA and obtained the ability to start methadone onsite. Harm reduction

Harm reduction specialists were onsite to provide education, naloxone, and rapid HIV tests.Safe injection supplies were offered to patients at the time of discharge.
}

Even more CRU patients carried a diagnosis of some form of mental illness than those who reported active substance use. Many of these patients had been receiving mental health treatment prior to admission. Many brought psychotropic medications with them and continued them in the CRU, or CRU clinicians were able to prescribe medications based on documentation in their medical record. Patients who reported acute symptoms or exhibited signs of mental distress were evaluated by clinicians in the CRU. If expert input was needed, BMC psychiatrists were available for telehealth consults, 
and clinical social workers were available to provide mental health counseling in person or via telehealth.

Acute exacerbations of non-COVID-related medical or mental health issues were addressed, but there was less focus on managing chronic health problems.

\section{PROGRAM EVALUATION}

Admissions to the CRU were tracked in the BMC hospital admissions system and in the Epic Electronic Health Record. A retrospective analysis of these data was performed after the CRU closed on June 4, 2020, including source of referrals, patient demographics, presence of COVID-19 symptoms, cooccurring mental health or substance use problems, length of stay, whether patients had acute complications, transfers to acute care settings, and clearance from isolation precautions. Reported data were based on simple counts via clinical chart review. This study was approved by the Boston University Medical Campus Institutional Review Board (H-40286).

Between April 9 and June 4, 2020, 226 patients were treated in the CRU, with an average length of stay of 7.3 days. Most (146) were referred from BMC inpatient or ED, and the rest from other hospitals, shelters, and testing sites. Seventy-two percent of admitted patients were male; 39\% identified as Black, 11\% Hispanic/LatinX (Table 2). Seventy-nine percent had $\geq 1$ psychiatric diagnosis, and $42 \%$ reported active substance use (Table 3). There were no deaths. At least 7 patients experienced a non-fatal overdose and $5 \%$ of patients developed serious complications of COVID-19 (Table 4). Seven percent of patients left prior to being medically cleared from isolation, but $1 / 3$ of those who left against medical advice subsequently returned. After completing isolation, 24 patients

Table 2 Patient Characteristics During Treatment at COVID Recuperation Unit, Massachusetts $2020(n=226)$

\begin{tabular}{ll}
\hline Baseline characteristics & Full cohort, $\boldsymbol{n} \mathbf{( \% )}$ \\
\hline Race/ethnicity & \\
Black, non-Hispanic & $88(38.9 \%)$ \\
White, non-Hispanic & $71(31.4 \%)$ \\
Hispanic or Latinx & $24(10.6 \%)$ \\
Other & $3(1.4 \%)$ \\
Unknown & $40(17.7 \%)$ \\
Psychiatric comorbidities ${ }^{*+}$ & $86(38.1 \%)$ \\
Depression & $78(34.5 \%)$ \\
Anxiety & $42(18.6 \%)$ \\
Post Traumatic Stress Disorder & $37(16.4 \%)$ \\
Bipolar disorder & $26(11.5 \%)$ \\
Schizophrenia/schizoaffective disorder & $13(5.8 \%)$ \\
Brain injury & $64(28.3 \%)$ \\
Active substance use at time of admission ${ }^{*+}$ & $43(19.0 \%)$ \\
Alcohol & $43(19.0 \%)$ \\
Opioids & $4(1.8 \%)$ \\
Cocaine/crack & $3(1.3 \%)$ \\
Methamphetamines &
\end{tabular}

*Not mutually exclusive

+ Reported in electronic health record or medical provider's assessment
Table 3 Baseline Psychiatric Diagnoses and Active Substance Use Status in COVID Recuperation Unit, Massachusetts $2020(n=226)$

\begin{tabular}{ll}
\hline \hline Baseline diagnoses & $\begin{array}{l}\text { Full cohort, } \boldsymbol{n} \\
(\mathbf{\%})\end{array}$ \\
\hline Patients with psychiatric diagnoses & $179(79.2 \%)$ \\
Patients with two or more psychiatric diagnoses & $86(38.0 \%)$ \\
Patients with active drug use & $94(41.6 \%)$ \\
Patients who actively use more than one drug & $40(17.7 \%)$ \\
Patients who have at least one psychiatric diagnosis & $68(30.1 \%)$ \\
and actively use at least one substance & \\
\hline
\end{tabular}

*Excludes tobacco and marijuana use

were discharged to SUD or mental health programs, 28 to stay with family members, and the rest to shelters (data not shown)

The CRU preserved inpatient beds for patients who needed acute care in at least two ways. ${ }^{11}$ First, the CRU admitted patients who did not need acute care but could not be discharged from the ED due to risk of contagion. Second, the CRU admitted patients who were medically ready for discharge after hospitalization but still needed isolation.

\section{DISCUSSION}

\section{Challenges}

Although the development and implementation of the CRU were generally quite successful, the program faced a number of challenges.

Staffing. Staff were recruited from various settings and did not work full time on the unit. Many did not have experience working with PEH or managing mental illness and substance use. This made it difficult to create a consistent culture of harm reduction free of stigma in the CRU.

Determining Appropriate Discharge Criteria. Criteria for clearance from isolation precautions changed frequently due to evolving Center for Disease Control and Prevention (CDC) recommendations and shortage of COVID tests. Swab-based discharge criteria turned out to be impractical, and inconsistent

Table 4 Discharge/Transfer Events to Boston Medical Center for Medical/Psychiatric Complications from COVID Recuperation Unit, Massachusetts $2020(n=226)$

\begin{tabular}{ll}
\hline \hline Rationale for evaluation & $\begin{array}{l}\text { Full cohort, } \boldsymbol{n} \\
(\mathbf{\%})\end{array}$ \\
\hline $\begin{array}{l}\text { Medical evaluation - apparent exacerbation of } \\
\text { COVID-19 symptoms }\end{array}$ & $11(4.9 \%)$ \\
$\quad \begin{array}{l}\text { Acute respiratory failure and/or low oxygen } \\
\text { saturation }\end{array}$ & $5(2.2 \%)$ \\
$\quad$ Acute cardiac issues & $4(1.8 \%)$ \\
Coagulation issues & $1(0.4 \%)$ \\
$\quad$ Renal issues & $1(0.4 \%)$ \\
Medical evaluation-apparently unrelated to & $9(4.0 \%)$ \\
COVID-19 infection & $7(3.1 \%)$ \\
\hline Psychiatric evaluation &
\end{tabular}


with the approach taken by partner organizations, since many patients' swabs remained positive for weeks; and so discharge criteria were revised to align with the CDC's 10-day symptom-based discharge. ${ }^{12}$

Predicting Demand for Beds. Demand was driven by the curve of the epidemic, but also by two other factors. First, COVID-19 testing was intermittent in PEH. When testing occurred, large groups of patients were suddenly identified who needed admission for isolation and quarantine. ${ }^{4} \mathrm{~A}$ second challenge was the tremendous variability in the prevalence of COVID-19 infection that was found during testing, ranging from 37 to $0 \%$ over the course of 8 weeks. This variability meant that daily admissions ranged from 0 to 17 , making it difficult to predict staffing needs.

Patient Use of Drugs/Harm Reduction. Within the CRU, it was challenging to fully implement a harm reduction approach. There was disagreement over the appropriate approach, with some medical staff advocating for harm reduction, while security personnel as well as some medical and operations staff and administration officials expressed concerns regarding safety if patients used non-prescribed substances on hospital grounds and public perception regarding laws that prohibit supervised injection sites. A compromise included distribution of sterile syringes at the time of discharge (rather than admission), and a policy of having clinicians, rather than security personnel, address use of substances.

Caring for Patients with Serious Mental Illness. Serious mental illness was common, and initially, staff were uncomfortable caring for patients who were paranoid, actively hallucinating, or delusional. Later, counselors and social workers became available to assist on the unit, and psychiatric consultations were available via telehealth, which lessened staff concerns. The CRU filled an important need in the community by providing care for COVID-infected patients with serious mental illness. ${ }^{13}$

\section{Lessons Learned}

BMC expanded its role as a safety-net hospital to provide care for COVID-infected PEH and utilized the opportunity to initiate treatment for SUDs for many patients, as well as caring for patients with severe psychiatric disorders. Rapid deployment of services in this emergency was achieved through hospital and Commonwealth coordination, and relaxation of regulations to allow speed and efficiency. Community partnerships were key factors in our success.

The CRU helped BMC avoid exceeding hospital bed capacity during the epidemic surge. ${ }^{11}$ Lower-acuity bed capacity in the CRU provided a vitally important release mechanism to allow BMC to reserve inpatient beds for patients with critical needs. Other cities that are currently being affected by the COVID-19 surge should consider similar programs that increase lower-acuity bed capacity for vulnerable populations.

If we had known before we implemented the CRU what we later learned, we would have done a few things differently. First, it is difficult to persuade patients who have been actively engaged in substance use to remain in an isolation setting, and it is also difficult to manage ongoing substance use in a hospital-like unit. It would have been helpful to develop consensus among clinical and administrative leaders in advance about how to address and balance these issues. Second, we would have implemented weekly virtual all-staff meetings to share evolving information and to provide ongoing training for staff. And third, it was painful to discharge patients back to shelters and to the street. The reality of the housing situation in Boston is that there is no immediately accessible permanent supportive housing. However, we wish that we had found other ways of helping to meet patients' needs at the time of discharge, such as connecting them with food resources.

One final lesson learned is that the experience of creating the CRU has shown us that we can provide safe and effective medical respite for PEH, and has inspired us to explore the implementation of similar services beyond the COVID-19 pandemic.

Acknowledgments: Special thanks to Kerry Sachs, Sarah Arbelaez, Deeanna Haidar, Deanna Faretra, Erin Nahrgang, Jessica Taylor, Jessica Kehoe, Simeon Kimmel, Zoe Weinstein, Bob Biggio, Norm Stein, Jeffrey Kalish, Joy Vreeland, Ravin Davidoff, Alastair Bell, and Kate Walsh, and to all of the physician volunteers who staffed the CRU.

Corresponding Author: Miriam Komaromy, MD; Grayken Center for Addiction, Boston Medical Center, Boston, MA, USA (e-mail: Miriam. Komaromy@bmc.org).

Funding Miriam Komaromy is partially supported by NIMH (UO1MH121954) and receives salary support for curriculum development from the American Society of Addiction Medicine. Miriam Harris is supported by the Research in Addiction Medicine Fellowship NIDA (R25DA033211). Joshua A. Barocas is supported by NIDA 1K01DA051684-01. Glorimar Ruiz-Mercado is partially funded by a Frontlines of Communities in the United States (FOCUS) grant from Gilead Sciences that supports HIV, hepatitis $C$ virus, and hepatitis $B$ virus screening and linkage to care.

\section{Compliance with Ethical Standards:}

Disclaimer: Gilead Sciences had no role in the development of this paper.

Conflict of Interest: The authors report no other relevant conflicts of interest.

\section{REFERENCES}

1. Tobolowsky FA. COVID-19 Outbreak Among Three Affiliated Homeless Service Sites - King County, Washington, 2020. MMWR Morb Mortal Wkly Rep. 2020;69. https://doi.org/10.15585/mmwr.mm6917e2.

2. Mosites E. Assessment of SARS-CoV-2 Infection Prevalence in Homeless Shelters - Four U.S. Cities, March 27-April 15, 2020. MMWR Morb Mortal Wkly Rep. 2020;69. https://doi.org/10.15585/mmwr. mm6917el. 
3. Baggett TP, Keyes H, Sporn N, Gaeta JM. Prevalence of SARS-CoV-2 Infection in Residents of a Large Homeless Shelter in Boston. JAMA. 2020;323(21):2191-2192. https://doi.org/10.1001/jama.2020.6887.

4. Gaeta JM, De Las Nueces D, Munson DG, Barocas JA, Walsh KE. Case 21-2020: A 66-Year-Old Homeless Man with Covid-19. N Engl J Med. 2020;383(2):170-178. https://doi.org/10.1056/NEJMcpc2002421.

5. Tsai J, Wilson M. COVID-19: a potential public health problem for homeless populations. Lancet Public Health. 2020;5(4):e186-e187. https://doi.org/10.1016/S2468-2667(20)30053-0.

6. Fazel S, Khosla V, Doll H, Geddes J. The Prevalence of Mental Disorders among the Homeless in Western Countries: Systematic Review and MetaRegression Analysis. PLoS Med. 2008;5(12):e225. https://doi.org/10. 1371/journal.pmed.0050225.

7. Volkow ND. Collision of the COVID-19 and Addiction Epidemics. Ann Intern Med. 2020;173(1):61-62. https://doi.org/10.7326/M20-1212.

8. Champagne-Langabeer T, Swank MW, Langabeer JR. Routes of non traditional entry into buprenorphine treatment programs. Subst Abuse Treat Prev Policy. 2020;15(1):6. https://doi.org/10.1186/s13011-0200252-z.

9. Public Health Commission. First case of 2019 Novel Coronavirus confirmed in Boston. Boston.gov. Published February 4, 2020. Accessed July 15, 2020. https://www.boston.gov/news/first-case-2019-novel-coronavirus-confirmed-boston
10. Bureau of Health Care Safety and Quality. Circular Letter DHCQ 20-03701 Authorization and Guidelines for Use of Alternate Space for Treatment of Ambulatory Patients Presenting with possible COVID-19. Published online March 6, 2020.

11. Hsu HE. Race/Ethnicity, Underlying Medical Conditions, Homelessness, and Hospitalization Status of Adult Patients with COVID-19 at an Urban Safety-Net Medical Center - Boston, Massachusetts, 2020. MMWR Morb Mortal Wkly Rep. 2020;69. https://doi.org/10.15585/mmwr. mm6927a3.

12. CDC. Coronavirus Disease 2019 (COVID-19). Centers for Disease Control and Prevention. Published February 11, 2020. Accessed July 15, 2020. https://www.cdc.gov/coronavirus/2019-ncov/hcp/disposition-hospitalized-patients.html

13. Druss BG. Addressing the COVID-19 Pandemic in Populations With Serious Mental Illness. JAMA Psychiatry. Published online April 3, 2020. https://doi.org/10.1001/jamapsychiatry.2020.0894.

Publisher's Note: Springer Nature remains neutral with regard to jurisdictional claims in published maps and institutional affiliations. 